\title{
The genetic variation of Angiostrongylus cantonensis in the People's Republic of China
}

\author{
Shan LV $v^{1,2,3^{*}}$ D, Yi Zhang ${ }^{1}$, Peter Steinmann ${ }^{2,3}$, Jürg Utzinger ${ }^{2,3}$ and Xiao-Nong Zhou ${ }^{1}$
}

\begin{abstract}
Background: The People's Republic of China (P.R. China) is the presumptive home range of the rat lungworm Angiostrongylus cantonensis, a major aetiological agent of human eosinophilic meningitis. We present a study of the genetic variation of $A$. cantonensis in P.R. China. Our aim was to deepen the current knowledge pertaining to its origin and global spread from a molecular perspective.

Methods: Adult A. cantonensis were collected in the frame of a national survey and identified based on morphological criteria. Polymerase chain reaction (PCR) was employed to amplify the target DNA sequences (cytochrome c oxidase subunit I (cox1), nicotinamide adenine dinucleotide dehydrogenase subunit 1 (nad1) and internal transcribed spacer (ITS)). The PCR product of cox 1 was directly submitted to sequencing, while clone sequencing was used for nad1 and ITS. The identity of the samples was verified by comparing the sequences to those of accepted A. cantonensis specimens. The specific composition of substitutions in each gene was analysed, and the genotypes were compared based on the complete cox1, nad1 and ITS genes.
\end{abstract}

Results: We characterised the complete mitochondrial genes cox 1 and nad 1 of 130 specimens and obtained 357 nuclear sequences containing two complete ITS (ITS1 and ITS2) and 5.8S rRNA of the same samples. All specimens were genetically confirmed as A. cantonensis. Two major groups (i.e. I and II) were identified according to the phylogeny of cox 1 sequences. Group I could be further categorised into six distinct clades. Almost half of the specimens (47.7\%) belong to the clade la and $22.3 \%$ to the group II. The former was widely distributed across the study region. A variable number of repeat units in three microsatellites was observed, resulting in considerable length variation in ITS. Intragenomic variation of ITS sequences was found in a large proportion of the samples. Genotyping showed a striking difference between mitochondrial DNA and ITS.

Conclusions: Our results demonstrate that A. cantonensis is the only rat lungworm species in P.R. China and shows high genetic diversity. Results of diversity and genotyping of $A$. cantonensis can be impacted by the sequencing strategy and biomarker. Although ITS may be a valuable marker for interspecific identification, it is not suitable for studying the intraspecific variation of $A$. cantonensis due to its high intragenomic variation and current challenges for direct sequencing.

Keywords: Angiostrongylus cantonensis, Genetics, cox1, nad1, Internal transcribed spacer, People's Republic of China

\footnotetext{
*Correspondence: Ivshan000@126.com

${ }^{1}$ National Institute of Parasitic Diseases, Chinese Center for Disease Control

and Prevention, Shanghai 200025, People's Republic of China

${ }^{2}$ Swiss Tropical and Public Health Institute, P.O. Box, CH-4002 Basel,

Switzerland

Full list of author information is available at the end of the article
} 


\section{Multilingual abstracts}

Please see Additional file 1 for translations of the abstract into the six official working languages of the United Nations.

\section{Background}

The rat lungworm Angiostrongylus cantonensis is an important aetiological agent of human eosinophilic meningitis [1]. The life cycle of $A$. cantonensis involves rats as definitive host and mollusks as intermediate host. Humans acquire infections mainly through the consumption of undercooked snails that harbour infective larvae. Humans are not permissive hosts. Larvae mainly dwell in the vessels of the central nervous system (CNS) and only rarely migrate to the pulmonary arteries [2]. Inflammation in the CNS is the proximate cause of morbidity due to an infection with $A$. cantonensis. Severe headache and paresthesia are the most common symptoms. Light infections are usually self-limiting, but heavy infections can be fatal, with young children at highest risk [3]. Globally, more than 3000 human cases have been reported [1].

There is consensus that $A$. cantonensis originated from the southern regions of the People's Republic of China (P.R. China) [4] or the Indochinese peninsula [5]. In the wake of the global spread of rats (Rattus rattus and $R$. norvegicus) and certain snail species (e.g. Achatina fulica and Pomacea spp.) [6], A. cantonensis is spreading across the tropics and subtropics. Thus far, over 30 countries have reported local $A$. cantonensis transmission [1], and recent reports suggest a further expansion [7-12].

Molecular evidence can deepen the understanding of the global spread of $A$. cantonensis. Two molecular markers (i.e. cytochrome c oxidase subunit I (cox1) and internal transcribed spacer (ITS)), have been employed to elucidate the phylogeny of $A$. cantonensis [7, 13-18]. In general, cox 1 proved to be a particularly useful marker for phylogenetic inference, whereas ITS is more valuable for interspecies differentiation.

A. cantonensis was first reported from Guangzhou, P.R. China, in 1935 [19]. A probably identical nematode was described in Taiwan two years later [20]. These early observations indicate that the South of P.R. China and offshore islands constituted the native range of $A$. cantonensis. However, the full extent of the endemic area was not revealed until recently $[3,21]$ and the genetic diversity of A. cantonensis remains to be fully elucidated $[14,18,22]$. Insights into the genetic diversity of $A$. cantonensis in P.R. China will contribute to the existing knowledge and be helpful to clarify the global spread of the parasite. The aim of this study was to present the genetic variation of $A$. cantonensis in P.R. China to deepen the current knowledge-base pertaining to its origin and global spread from a molecular perspective.

\section{Methods}

\section{Collection of $A$. cantonensis specimens and total DNA preparation}

A. cantonensis specimens were collected in the frame of the first national survey of the distribution of angiostrongyliasis in the mainland of P.R. China, which was implemented in 2006 and 2007 [21] (Fig. 1). Wild rats ( $R$. norvegicus, $R$. rattus and $R$. flavipectus) and mollusks were collected and examined for the presence of Angiostrongylus spp., based on morphological criteria of adult worms. In localities where adult worms could not be discovered in rats, the larvae were collected from infected Pomacea spp. and/or Achatina fulica snails. SpragueDawley (SD) rats were infected with pools of 20-50 larvae in laboratory. Adult worms were then collected from the rats six weeks post-infection and identified morphologically. All adult Angiostrongylus worms were kept in $75 \%$ ethanol pending further genetic identification. For comparison, A. cantonensis DNA specimens from Thailand were kindly provided by Dr. Praphathip Eamsobhana from Mahidol University, using a Flinders Technology Associates (FTA) card [16].

A. cantonensis specimens were individually washed three times using phosphate buffered saline (PBS) and placed into clean $1.5 \mathrm{ml}$ tubes. Worms were then cut into small pieces and incubated with sodium dodecylsulphate/proteinase $\mathrm{K}$ at $56{ }^{\circ} \mathrm{C}$, pH 7.4, for 4-6 h [23]. The suspension was centrifuged and the supernatant transferred into another tube for extraction with phenol/ chloroform/isoamyl alcohol (v:v:v $=25: 24: 1)$. The DNA pellet was suspended in $30-50 \mu \mathrm{H} \mathrm{H}_{2} \mathrm{O}$ and kept at $-20{ }^{\circ} \mathrm{C}$ pending analysis.

\section{Polymerase chain reaction (PCR) and sequencing}

The primers targeting two mitochondrial genes, nad 1 and cox 1 , were designed according to the complete mitochondrial genome of A. cantonensis (GQ398121) (Table 1). To obtain the full gene sequence of $\operatorname{cox} 1$, two overlapping fragments were amplified. We employed universal primers for the complete sequence of two nuclear ITS (i.e. ITS1 and ITS2) and 5.8S ribosomal RNA genes [18]. PCR was performed in $50 \mu \mathrm{l}$ with $1.5 \mathrm{mM}$ of $\mathrm{MgCl}_{2}, 10 \mu \mathrm{M}$ of each primer, $25 \mu \mathrm{l} 2 \times \mathrm{Taq}$ buffer, $0.2 \mathrm{mM}$ of each dNTPs, $2.5 \mathrm{U}$ of Taq DNA polymerase and $1 \mu \mathrm{l}$ of DNA sample as follows: $94{ }^{\circ} \mathrm{C}$ for 5 min, 35 cycles at $94{ }^{\circ} \mathrm{C}$ for $60 \mathrm{~s}$, around $48-55^{\circ} \mathrm{C}$ for $60 \mathrm{~s}$, and $72{ }^{\circ} \mathrm{C}$ for $60-90 \mathrm{~s}$, followed by $72{ }^{\circ} \mathrm{C}$ for $10 \mathrm{~min}$ for the final extension.

PCR products of cox 1 were directly sequenced. Since the products of nad1 and ITS could not be sequenced due to the heterogeneity caused by PCR incorporated error in the regions of poly-adenine or thymine in nad1 or within-individual heterogeneity in ITS, they were instead harvested from the gel over mini-spin columns 


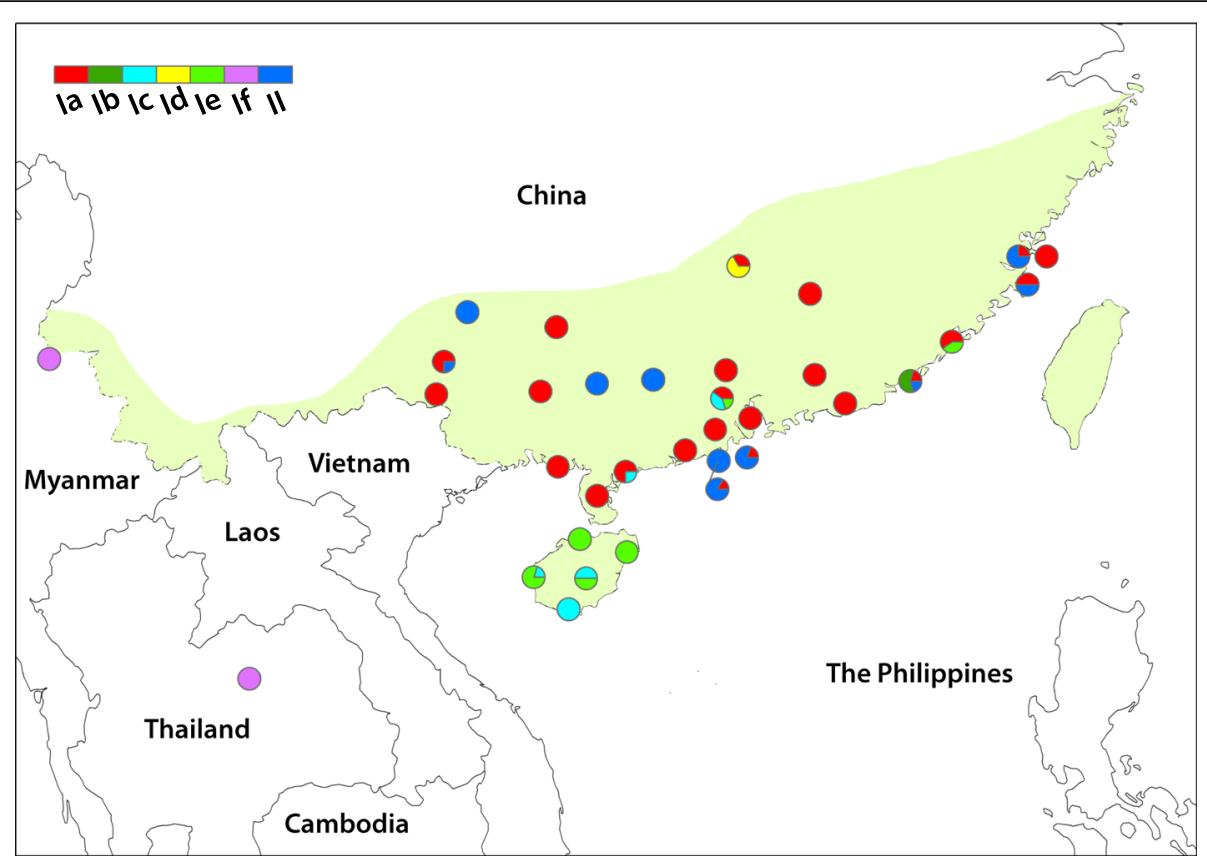

Fig. 1 Geographical distribution of cox1 clades of Angiostrongylus cantonensis in P.R. China. Two groups, i.e. I and II, are shown. Six clades are further distinguished in group I ( $\mathrm{l}, \mathrm{lb}, \mathrm{lc}, \mathrm{ld}$, le and If). The current endemic area of A. cantonensis in P.R. China is indicated by shading

(Axygen; Union, USA). The purified PCR products were ligated into $\mathrm{pGEM}^{\oplus} \mathrm{-T}$ Easy vectors with the LigaFast ligation system (Promega; Shanghai, P.R. China). The plasmid vector with the target fragment was transformed into JM109 or DH5 $\alpha$ Escherichia coli, according to the manufacturer's instructions. Positive clones were then subjected to sequencing using the dideoxynucleotide termination method. One clone of the nad1 gene and three clones of the ITS gene from each specimen were selected for sequencing. All fragments were determined by double-directional sequencing.

\section{Data from GenBank}

Previous studies identified 13 unique clades of the $\operatorname{cox} 1$ gene of $A$. cantonensis [24]. The typical sequences available in GenBank were utilized to infer the phylogeny. The access numbers are indicated in Fig. 3. The complete cox1 sequence of $A$. malaysiensis (KT947979), A. costaricensis (GQ398122), A. vasorum (JX268542), Metastrongylus salmi (GQ888715), M. pudendotectus (GQ888714) and Aelurostrongylus abstrusus (JX519458) were used as outgroups.

\section{Data analysis of mitochondrial genes}

The sequences of each target gene were aligned using ClustalX version 2.0 [25] and trimmed using BioEdit version 7 . The truncated sequences were then presented to DnaSP version 5 [26] to collapse into unique haplotypes for subsequent phylogenetic analysis. Polymorphic sites and DNA polymorphism were analysed by DnaSP version 5 . The haplotype diversity $(\mathrm{Hd})$, and nucleotide diversity $(\mathrm{Pi})$ were estimated.

Before reconstructing the phylogenetic tree, the bestfitting nucleotide substitution model was determined by

Table 1 Primers used in the present study to determine the genetic structure of A. cantonensis in P.R. China

\begin{tabular}{|c|c|c|c|}
\hline Gene & Primer & Primer sequence & Annealing temperature \\
\hline \multirow[t]{2}{*}{ nad1 } & ND1_F & 5'-GATTTAGTTATTCTTGTTG-3' & $48^{\circ} \mathrm{C}$ \\
\hline & ND1_R & $5^{\prime}-C C A A C A A A A A C A C A T C T A A C-3^{\prime}$ & \\
\hline \multirow[t]{4}{*}{$\operatorname{cox} 1$} & COI_F1 & 5'-GGTGATTATAATGTTTAATG-3' & $53^{\circ} \mathrm{C}$ \\
\hline & COI_R1 & 5'-CGTAGGAACCGCAATAAC-3' & \\
\hline & COI_F2 & 5'-TATGGTTTATGCTATITTAAG-3' & $53^{\circ} \mathrm{C}$ \\
\hline & COI_R2 & 5'-GGCACTACACAACGATTATC-3' & \\
\hline \multirow[t]{2}{*}{ ITS } & ITS_F & 5'-GTAGGTGAACCTGCGGAAGGATCATT-3' & $55^{\circ} \mathrm{C}$ \\
\hline & ITS_S & 5'-TTAGTTTCTTTTCCTCCGCT-3' & \\
\hline
\end{tabular}


jModeltest version 0.1.1 [27]. Bayesian inference was conducted in MrBayes version 3.1 [28] until the average standard deviation of split frequencies were below 0.01 , and the potential scale reduction factor was reasonably close to 1.0 for all parameters. Neighbour-joining and maximum parsimony estimates were performed in Mega version 5.05 [29] with bootstrap testing (1000 replicates). The Bayesian consensus trees were summarised and visualised by Mesquite version 2.75 [30]. The geographical distribution of the clades of the cox 1 gene was mapped with the geographical information system ArcInfo 9.2.

\section{Data analysis of ITS}

The variable sites of mitochondrial and ITS genes were plotted to compare their validity for phylogeny. At each variable site, the parsimony-informative and -uninformative sites were distinguished, and the proportion of nucleotide substitutions across all sequences was calculated.

A few microsatellites were identified during the initial alignment. Three microsatellites, which showed considerable variation in the number of repeats, were extracted for further analysis. The types for each microsatellite were categorised and the different microsatellite combinations were counted. The distribution of these combinations among phylogenetic clades, determined by mitochondrial genes, was compared.

\section{Results}

\section{Variation of mitochondrial genes}

The $n a d 1$ and cox 1 genes of a total of 130 A. cantonensis specimens from 32 collection sites, representing the whole known endemic area of angiostrongyliasis in P.R. China, were successfully sequenced. Considerable variation was observed both in the sequences of nad1 and cox 1 . The number of haplotypes was 39 for cox 1 (Hd: 0.8114; Pi: 0.0284) and 75 for nad 1 (Hd: 0.9260; Pi: 0.0314), respectively. Among the 843 sites of the complete nad 1 gene and 1577 sites of the complete cox 1 gene, 171 variable sites were identified in each gene. Parsimony-informative sites accounted for the major proportion, i.e. $60.2 \%$ in $\operatorname{nad} 1(103 / 171)$ and $94.7 \%$ in $\operatorname{cox} 1(162 / 171)$. The latter is remarkably higher than the former (Fig. 2a and b). However, a considerable proportion of singlets in nad 1 was observed. It was probably caused by the sequencing strategy, i.e. clone sequencing; PCR incorporation errors got manifested when sequencing clones. Compound parsimony-informative sites, where three or four nucleotide types occurred simultaneously more than twice, were also noted at a frequency of 9 in cox 1 versus 3 in nad1. Additionally, many variable sites showed a similar proportion of nucleotide substitutions, denoted by the dot line in Fig. 2a and b, which indicated a high validity of the gene in distinguishing the clades.

The proteins cytochrome oxidase subunit I (COI) and NADH dehydrogenase subunit 1 (NADH1) were

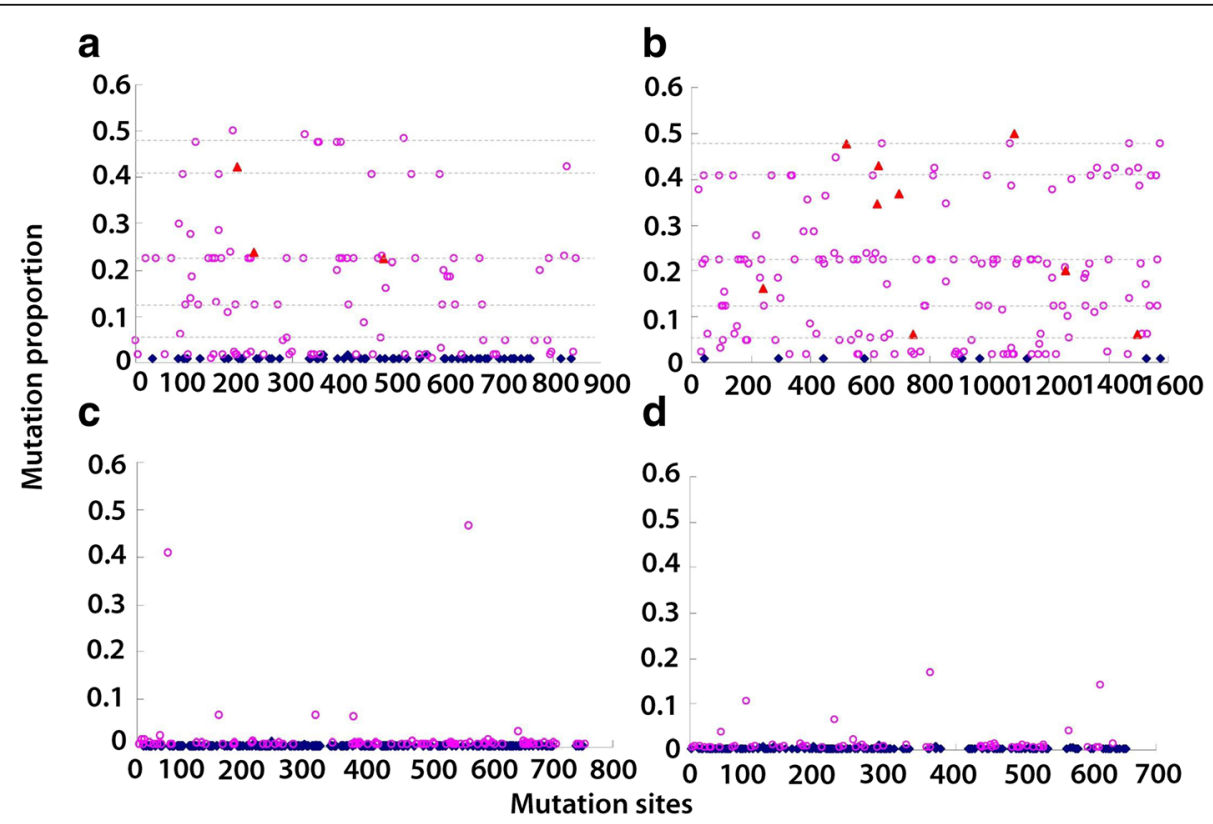

Fig. 2 Plots of variable sites by gens; nad1 (a), cox1 (b), ITS1 (c) and ITS2 (d). Three types of variations are shown (red diamond, compound parsimony-informative; pink circle, simple parsimony-informative; blue square, singleton). A compound parsimony-informative site refers to a position where three or four nucleotide types occur simultaneously more than twice in an alignment. The dot lines in (a) and (b) denote parsimony-informative sites with similar mutation proportions 
inferred to consist of 525 and 281 amino acids, respectively. Sixteen and 17 variable sites were identified in the presumed protein COI and NADH1, respectively. The corresponding synonymous variations are 18 and 73. Of note, a few variation sites in the protein sequence contain more than two amino acids, which resulted from mutations in a different codon position.

\section{Phylogeny based on cox 1 and mapping of clades}

In order to avoid false inference, the nad 1 data were excluded from the construction of the phylogeny. The tree inferred based on the complete sequence of cox 1 produced in the present study showed two distinct groups, namely I and II (Fig. 3). The group I can be further distinguished into six clades. Almost half of the specimens (47.7\%) fell into clade Ia and $22.3 \%$ into group II. The haplotypes from clades Ib and Id were limited to single collection sites (Fig. 1). All the 13 known clades of $A$. cantonensis identified by previous studies $[14,24]$ fell into the clades or groups identified in the present study.

\section{Variation of ITS}

Overall, 357 sequences containing complete ITS1, 5.8S rRNA and ITS2 were obtained from the $130 \mathrm{~A}$. cantonensis specimens. According to the structure of ribosomal RNAs of Caenorhabditis elegans and Metastrongylus [31, 32], the entire ITS1 and ITS2 genes were determined. The length of ITS1 ranged from $708 \mathrm{bp}$ to $743 \mathrm{bp}$ and that of ITS2 from $606 \mathrm{bp}$ to $654 \mathrm{bp}$. The difference in length within genes mainly resulted from the variable number of repeats in the microsatellites. However, deletion mutations of long fragments (7 bp in six sequences, $9 \mathrm{bp}, 22 \mathrm{bp}$ and $40 \mathrm{bp}$ in a single sequence, respectively) outside the microsatellites were also observed in ITS2.

A total of 300 variable sites were identified in ITS1 among 756 aligned sites, and 257 in ITS2 among 654 aligned sites. In contrast to mitochondrial genes, the proportion of parsimony-informative sites was only $32.7 \%$ in ITS1 $(98 / 300)$ and $30.0 \%$ in ITS2 (77/257). In addition, there were only 13 and 16 parsimonyinformative sites where the proportion of nucleotide substitution across all obtained sequences was higher than $1 \%$ in ITS1 and ITS2, respectively. It was

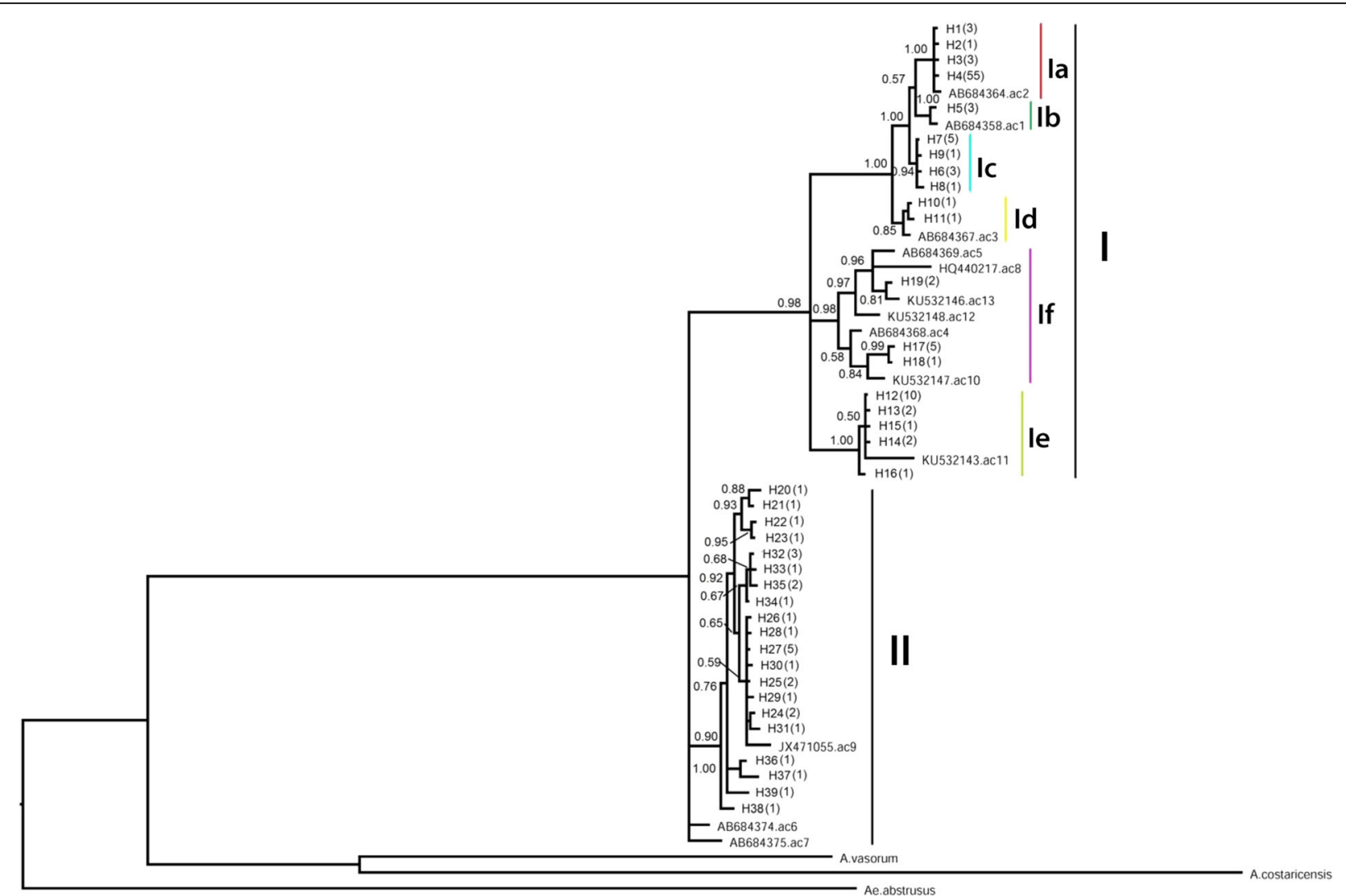

Fig. 3 Phylogenetic tree inferred by complete cox1 sequences. The posterior probability of Bayesian inference is marked at the branches. The number in brackets after the taxon name is the frequency of the haplotype. The letters with the vertical bar denote the clades. I and II denote the major groups 
significantly lower than those in mitochondrial genes (Fig. 2c and d).

Within-individual heterogeneity in the ITS was also noted. There were 90 specimens for which three complete ITS sequences from each individual specimen were obtained. Slightly less than a third of the specimens (30.0\%) showed three distinct haplotypes, determined by 29 parsimony-informative sites, whereas only $15.5 \%$ were homogeneous.

\section{Phylogeny based on ITS}

Bayesian inference and neighbour-joining did not resolve the in-depth topological relationship between each taxon well. The only consensus in the trees was that the clade If inferred based on mitochondrial genes was separated from any other taxa (Fig. 4). An attempt was made to draw inference by 29 parsimony-informative sites where the proportion of nucleotide substitution was higher than $1 \%$. Yet, this approach revealed similar results. Subsequently, the 29 parsimony-informative sites were used to explore the haplotypes. Seventy unique haplotypes were identified and 36 of them occurred with a frequency of 1 . The distribution of these haplotypes among the clades inferred by mitochondrial genes is shown in Fig. 5. A striking cross-transmission of haplotypes was observed among all clades, except for clade If. The number of shared haplotypes between each of the two clades increased with the growing pool of sequences.

\section{Variation of ITS microsatellites}

Twelve microsatellites were observed in the ITS1 and ITS2 sequences (Table 2). Three microsatellites (MS-5, 8 and 12) showed considerable variation in the number of repeat units, which was the major cause of the low success of direct sequencing of PCR products. The three microsatellites showed perfect repeats as well as imperfect repeats (Fig. 6). A total of 18 repeat types were found in MS-5. The other two microsatellites had 21 types, respectively. The diversity significantly increased when the three microsatellites were concatenated; there were 126 combinations among all the 357 ITS

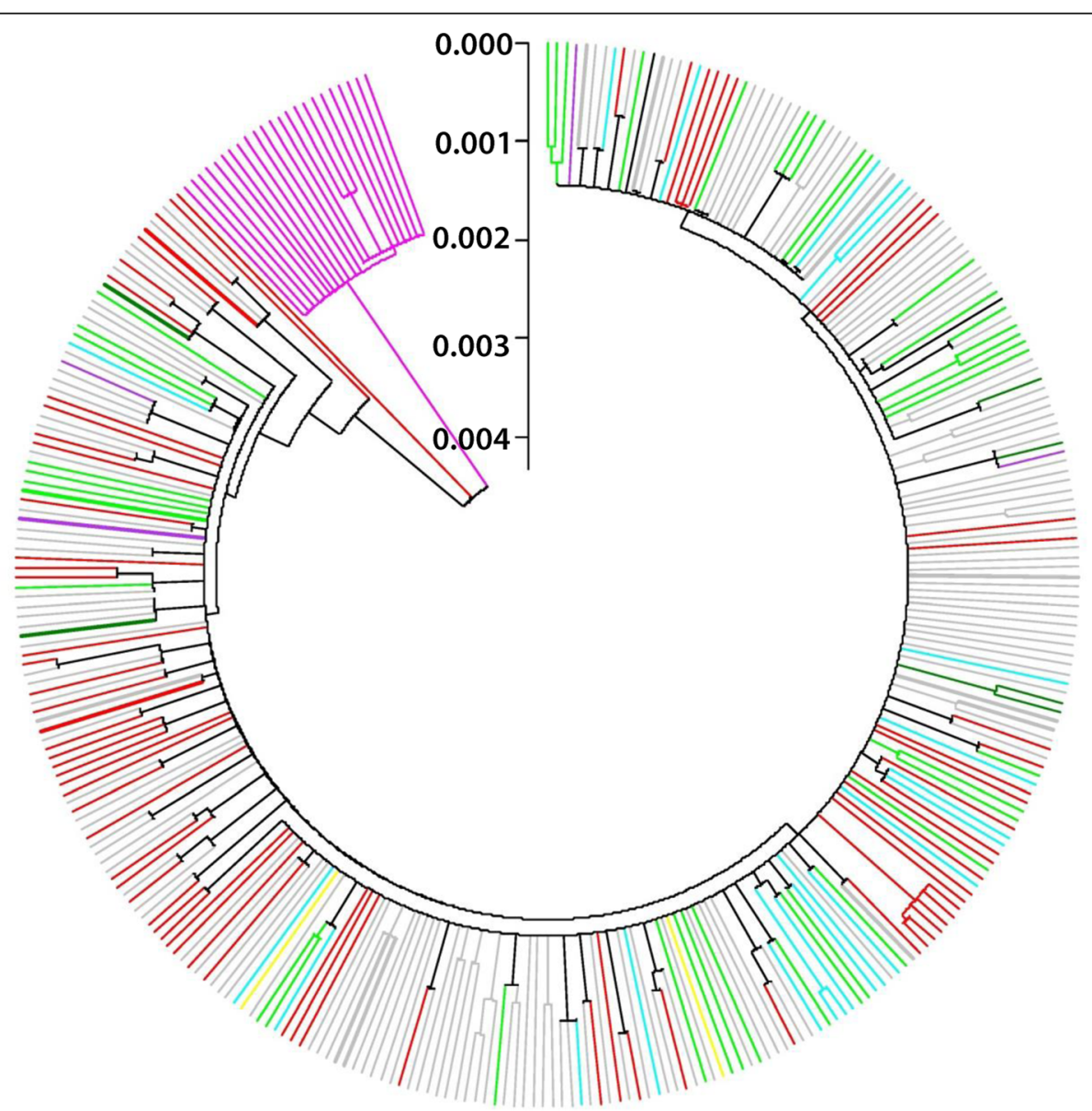

Fig. 4 Phylogenetic tree inferred by the complete ITS1, 5.8S and ITS2 sequence excluding the three length-variable microsatellites. The neighbour-joining method with the TrN + G model was used. Taxon name is hidden and the colors correspond to the clades in Fig. 3 . The bold branches indicate that the frequency is more than one 


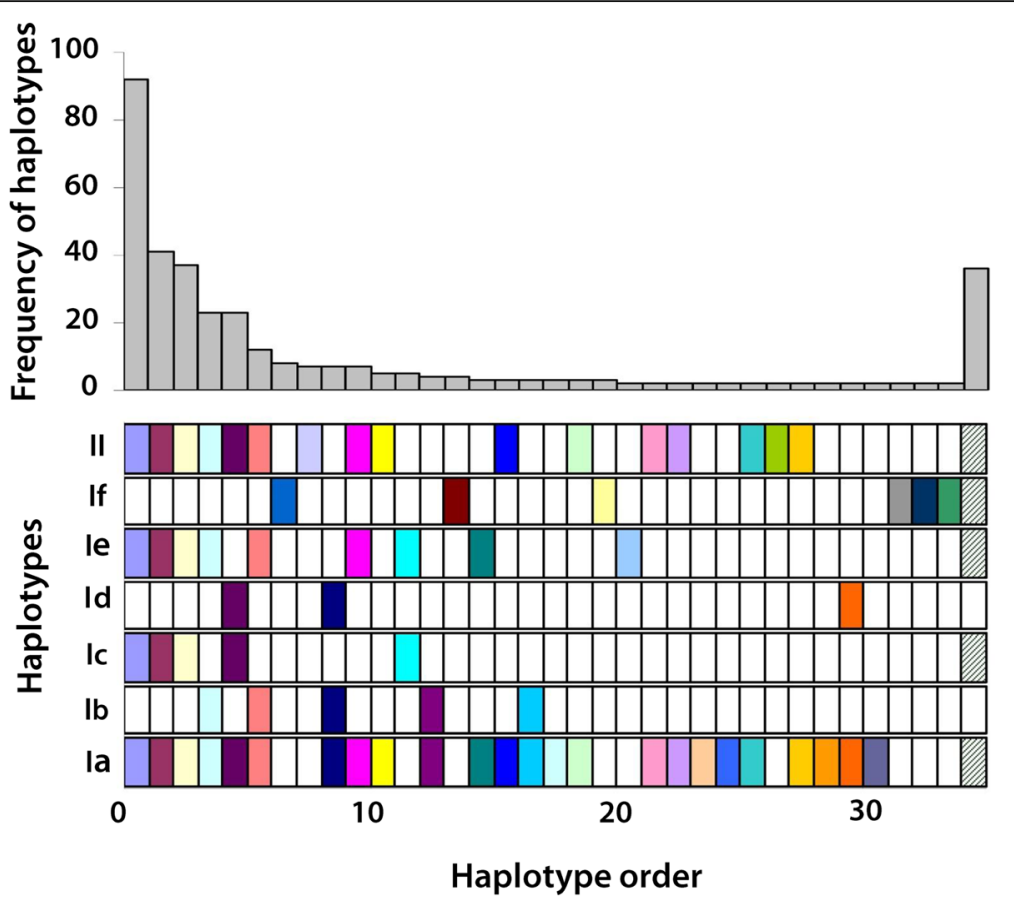

Fig. 5 Distribution of haplotypes determined by ITS among the clades inferred by cox 1 genes. The top chart is the frequency distribution of haplotypes, and the bottom bar chart indicates the distribution among different clades (denoted by letters). Each column represents one haplotype. The last column denotes all other haplotypes with a frequency of 1

sequences. However, the majority of them (86) occurred with a frequency of 1 .

It was also noted that some imperfect repeats could represent the special clades inferred by mitochondrial gene analysis. For example, $(\mathrm{CA})_{\mathrm{n}} \mathrm{TA}(\mathrm{CA})_{\mathrm{m}}$ in MS-5 and $(\mathrm{AT})_{\mathrm{n}} \mathrm{ACAC}(\mathrm{AT})_{\mathrm{m}}$ in $\mathrm{MS}-8$ only occurred in clade If. Furthermore, almost all the specimens in this clade showed the two imperfect repeats simultaneously.

With regard to microsatellites, we also explored the distribution of these combinations among the clades

Table 2 Microsatellites (MS) identified from the alignment of concatenated ITS1 and ITS2

\begin{tabular}{lllll}
\hline MS & Position & Length & Repeats & Sequence \\
\hline 1 & 73 & 3 & 3 & CGTCGTCGT \\
2 & 100 & 4 & 3 & GGTGGGTGGGTG \\
3 & 118 & 3 & 3 & TGATGATGA \\
4 & 255 & 2 & 3 & ACACAC \\
5 & 329 & 2 & Variable & (CA)n \\
6 & 401 & 2 & 3 & TGTGTG \\
7 & 478 & 2 & 4 & TGTGTGTG \\
8 & 727 & 2 & Variable & (AT)n \\
9 & 1178 & 4 & 4 & ATCGATCGATCGATCG \\
10 & 1282 & 2 & 3 & GTGTGT \\
11 & 1446 & 2 & 3 & ACACAC \\
12 & 1468 & 2 & Variable & (TG)n \\
\hline
\end{tabular}

inferred from mitochondrial genes (Fig. 7). Similarly, a notable cross-transmission occurred across all clades except for clade If, although the pattern was different from that derived from ITS. We also noted that clades Ia and II shared much more types than the other clades.

We observed considerable individual heterogeneity of microsatellite types. Two-third of the 90 samples, for which three complete ITS sequences were obtained, showed three distinct types, whereas only four samples (4.4\%) were homogeneous. The proportion $(66.7 \%)$ of individuals which had three distinct microsatellite types was much higher than that (30.0\%) of individuals which had three distinct ITS haplotypes. The conserved estimation in ITS haplotypes only using 29 parsimonyinformative sites might explain this lower proportion.

\section{Discussion}

The distribution of A. cantonensis in P.R. China is currently restricted to eight southern provinces [21]. As suggested by recent modelling work, it could change due to global warming, the further spread of invasive snail species and changing transmission dynamics [33]. We identified seven distinct clades based on the analysis of mitochondrial genes, which indicates a high diversity of A. cantonensis in its current presumptive home range. The major clades identified are Ia and II. Although A. malaysiensis was reported to occur in close proximity 

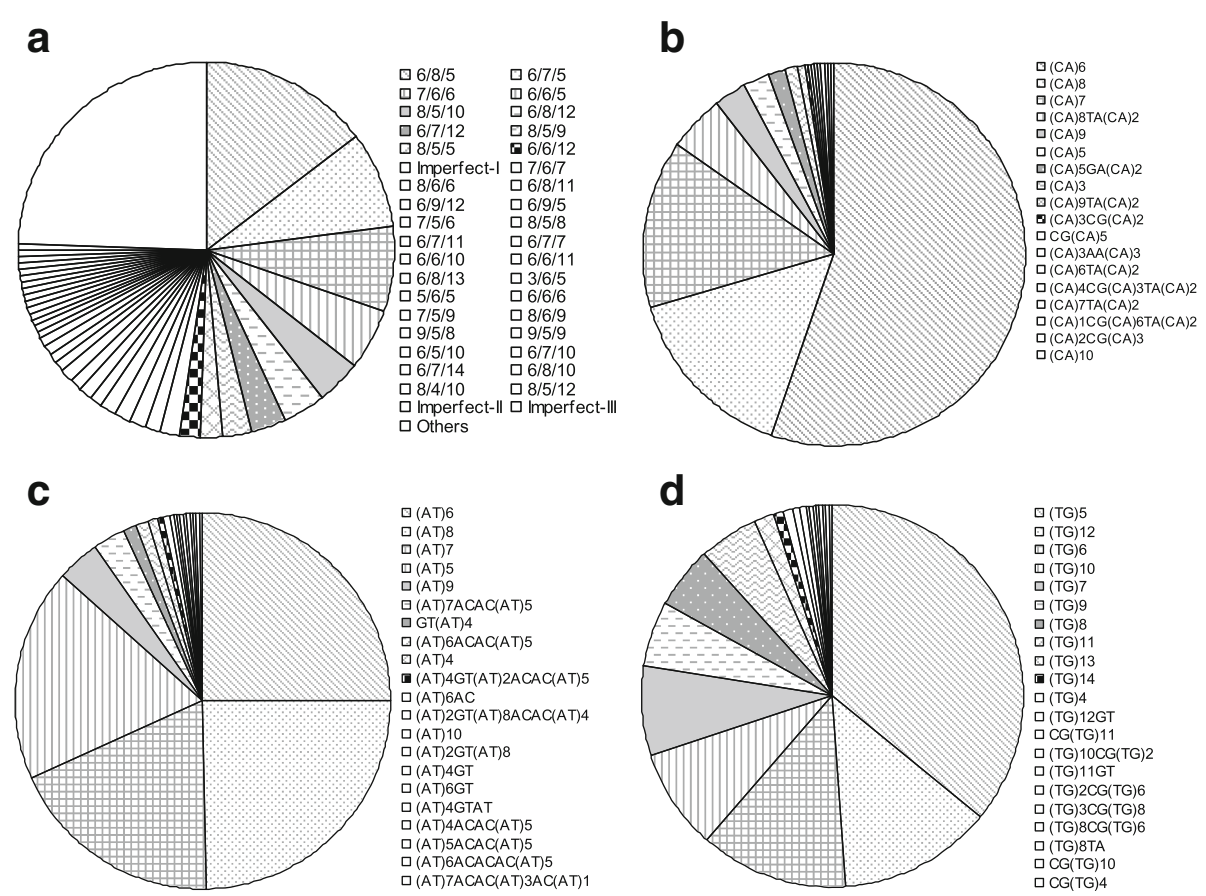

Fig. 6 Type of microsatellites; combination of three microsatellites (a), MS-5 (b), MS-8 (c) and MS-12 (d). Only 10 types with higher frequencies were denoted using different filled pies, with the others indicated by hollow pies. In Fig. $6 a$, the numbers separated by slash denotes the number of repeats in MS-5, 8 and 12, respectively

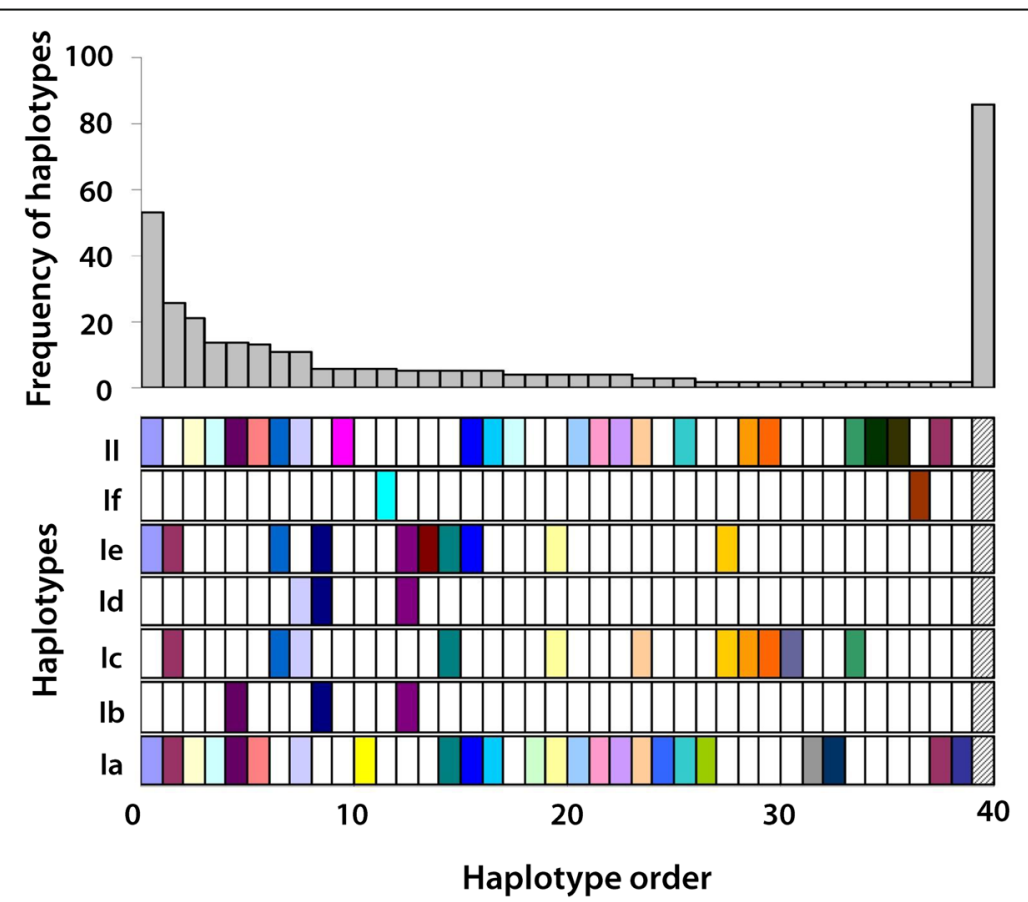

Fig. 7 Distribution of haplotypes determined by microsatellites among the clades inferred by mitochondrial genes. Only MS-5, 8 and 12 were included in the analysis. For other labels, the reader is referred to Fig. 5 
to the border with Vietnam [34], our results show that $A$. cantonensis is probably the only species endemic in mainland P.R. China. We further excluded the possibility of group II being a separate species, since there was a lack of essential differences in ITS sequences between this group and other clades. In addition, the wide cross-transmission of ITS genotypes and microsatellites implies the absence of any reproductive isolation. Furthermore, the genetic distance between group II and $A$. malaysiensis is long (results not shown due to a long-branch attraction). Instead, group II was much closer to the other clades of $A$. cantonensis.

Although clade If was found to be more closely related to clade Ia (the most common haplotype in P.R. China) than group II, nuclear genes indicated that clade If is a distinctive group. Indeed, there is no cross-transmission of haplotypes as inferred by ITS and microsatellites between clade If and any of the other clades. We speculate that geographical isolation is the root cause of this observation. The $A$. cantonensis specimens obtained from Yunnan Province fell into clade If, along with samples from Thailand. Thus, the samples collected from areas near the border to Myanmar might belong to the same transmission region as the Thai isolates. In contrast, we infer that group II probably was introduced into the mainland of P.R. China. Hybridization might play an important role in the nuclear similarity between group II and other presumptive native clades. However, the fate of original nuclear genetic characters of group II and/or native clades remains to be investigated.

We noted that the proportion of singleton variable sites is strikingly variable between nuclear genes and mitochondrial genes, and even among mitochondrial genes. First, there is a significantly higher proportion of singleton variable sites in nad1 (39.8\%) compared to cox1 (5.3\%). Second, singleton variable sites account for $67.3 \%$ of variable sites in ITS1 and $70.0 \%$ in ITS2, which is notably higher than in mitochondrial genes. Sequencing methods might explain the difference between $\operatorname{cox} 1$ and $n a d 1$, and further between mitochondrial genes and ITS sequences. Sequencing following cloning is sensitive to PCR-induced artifacts, and hence, might falsely increase the apparent diversity $[35,36]$. Therefore, direct sequencing of PCR product is recommended for future studies.

We found striking intragenomic or within-individual heterogeneity in both ITS sequences and microsatellites, which conflict with concerted evolution [37] but is consistent with previous studies [38, 39]. Although we reduced false diversity induced by cloning sequencing as mentioned above by excluding the sites where the proportion of nucleotide substitution was less than $1 \%$, our conserved estimate of within-individual heterogeneity using 29 parsimony-informative sites is still notable. This finding demonstrates that ITS is not a useful genetic marker for population genetic studies of $A$. cantonensis.

The length of ITS region in different nematode species shows considerable variation. For example, the ITS region of some representative species from Rhabditid and Cephalobid orders is between $275 \mathrm{bp}$ and $875 \mathrm{bp}$ long [40]. Our findings and previous studies indicate that the ITS length of the genus Angiostrongylus may be much longer than that of other nematodes [18, 41]. Indeed, the combination of ITS1 and ITS2 can be as long as $1377 \mathrm{bp}$, as described here. Like other members of the genus Angiostrongylus [42], A. cantonensis also shows microsatellites in the ITS regions and some of them are strikingly variable in the number of repeats, which is a challenge for direct sequencing of PCR products. Our findings suggest that the primer designation for ITS regions should avoid the microsatellites to potentially increase the success of direct sequencing of PCR products, which in turn will lower the number of artifacts induced by PCR.

\section{Conclusions}

We conclude that $A$. cantonensis is the only Angiostrongylus species in the study area. Our results show a high diversity of $A$. cantonensis in mitochondrial genes, which is helpful to elucidate the global spread from a molecular perspective. We observed intragenomic heterogeneity in ITS. Hence, ITS appears not to be an appropriate marker for genotyping of $A$. cantonensis. The sequencing strategy can considerably impact the haplotype diversity, and hence, clone sequencing cannot be recommended.

\section{Additional file}

Additional file 1: Multilingual abstracts in the six official working languages of the United Nations. (PDF $663 \mathrm{~kb}$ )

\section{Abbreviations \\ CNS: Central nervous system; cox1: The gene of cytochrome c oxidase subunit I; FTA: Flinders Technology Associates (card); Hd: Haplotype diversity; ITS: Internal transcribed spacer; MS: Microsatellites; nad1: The gene of nicotinamide adenine dinucleotide dehydrogenase subunit 1; PCR: Polymerase chain reaction; Pi: Nucleotide diversity; SD: Sprague-Dawley (rat)}

\section{Acknowledgements}

We thank Dr. Praphathip Eamsobhana for kindly providing the isolates of A. cantonensis from Thailand and Drs. Li-Sha Li, Zhuo-Hui Deng, Hong-Man

Zhang, Xi-Min Hu, Zheng-Xiang Li, Xiao-Jun Zeng and their colleagues for help with the field work, particularly the collection of $A$. cantonensis specimens.

\section{Funding}

This work was supported by International Development and Research Centre (IDRC; Canada) (grant no. 105509-00001002-023) and the International

Society of Infectious Diseases (small grant 2007 fall).

Availability of data and materials

Please contact the corresponding author for data requests. 


\section{Authors' contributions}

SL, YZ and XNZ conceived and designed the experiments. SL and YZ performed the study and experiments. SL analysed the data. $S L$ and $Y Z$ contributed reagents/materials/analysis tools. SL, YZ, PS, JU and XNZ wrote and revised the manuscript. All authors read and approved the final manuscript.

\section{Ethics approval and consent to participate}

The study protocol was approved by the institutional ethics committee of the National Institute of Parasitic Diseases, Chinese Center for Disease Control and Prevention (reference no. 2006030101). All animals were handled in strict accordance with good animal practice according to the Animal Ethics Procedures and Guidelines of the People's Republic of China.

\section{Consent for publication}

Not applicable.

\section{Competing interests}

Xiao-Nong Zhou is the Editor-in-Chief of Infectious Diseases of Poverty. The other authors declare that they have no competing interests.

\section{Author details}

${ }^{1}$ National Institute of Parasitic Diseases, Chinese Center for Disease Control and Prevention, Shanghai 200025, People's Republic of China. ${ }^{2}$ Swiss Tropical and Public Health Institute, P.O. Box, CH-4002 Basel, Switzerland. ${ }^{3}$ University of Basel, P.O. Box, $\mathrm{CH}-4003$ Basel, Switzerland.

\section{Received: 18 January 2017 Accepted: 26 July 2017}

\section{Published online: 01 September 2017}

\section{References}

1. Wang $Q P$, Lai $D H$, Zhu $X Q$, Chen $X G$, Lun ZR. Human angiostrongyliasis. Lancet Infect Dis. 2008;8:621-30.

2. LV S, Zhang $Y$, Steinmann $P$, Zhou $X N$, Utzinger J. Helminth infections of the central nervous system occurring in Southeast Asia and the Far East. Adv Parasitol. 2010;72:351-408.

3. LV S, Zhang Y, Steinmann P, Zhou XN. Emerging angiostrongyliasis in mainland China. Emerg Infect Dis. 2008;14:161-4.

4. Prociv P, Spratt DM, Carlisle MS. Neuro-angiostrongyliasis: unresolved issues. Int J Parasitol. 2000;30:1295-303.

5. Drozdz J, Gorecka T, Binh NH. The occurrence of nematodes of the subfamily Angiostrongylinae in Viet-Nam and the question of geographical origin of Parastrongylus cantonensis (Chen, 1935). Acta Parasitol. 1975;23:115-26.

6. Lowe S, Browne M, Boudjelas S, De Poorter M. 100 of the world's worst invasive alien species: a selection from the Global Invasive Species Database. Invasive Species Specialist Group (ISSG) a specialist group of the Species Survival Commission (SSC) of the World Conservation Union (IUCN); 2000: 12 p.

7. Foronda P, Lopez-Gonzalez M, Miquel J, Torres J, Segovia M, Abreu-Acosta N, Casanova JC, Valladares B, Mas-Coma S, Bargues MD, Feliu C. Finding of Parastrongylus cantonensis (Chen, 1935) in Rattus rattus in Tenerife, Canary Islands (Spain). Acta Trop. 2010;114:123-7.

8. Mugisha L, Bwangamoi O, Cranfield M. Angiostrongylus cantonensis and other parasites infections of rodents of Budongo Forest reserve, Uganda. Afr J Anim Biomed Sci. 2012;7:91-5.

9. Pincay T, Garcia T, Narvaez E, Decker O, Martini L, Moreira JM. Angiostrongiliasis due to Parastrongylus (Angiostrongylus) cantonensis in Ecuador. First report in South America. Trop Med Int Health. 2009;14(Suppl. 2):37.

10. Chikweto A, Bhaiyat MI, Macpherson CN, Deallie C, Pinckney RD, Richards C, Sharma RN. Existence of Angiostrongylus cantonensis in rats (Rattus norvegicus) in Grenada. West Indies Vet Parasitol. 2009;162:160-2.

11. Archer CE, Appleton CC, Mukaratirwa S, Hope KJ. The rat lung-worm Angiostrongylus cantonensis: a first report in South Africa. S Afr Med J. 2011;101:174-5.

12. Dard C, Piloquet JE, Qvarnstrom Y, Fox LM, M'Kada H, Hebert JC, Mattera D, Harrois D. First evidence of angiostrongyliasis caused by Angiostrongylus cantonensis in Guadeloupe, Lesser Antilles. Am J Trop Med Hyg. 2017;96:692-7.

13. Monte TC, Simoes RO, Oliveira AP, Novaes CF, Thiengo SC, Silva AJ, Estrela PC, Junior AM. Phylogenetic relationship of the Brazilian isolates of the rat lungworm Angiostrongylus cantonensis (Nematoda: Metastrongylidae) employing mitochondrial COI gene sequence data. Parasit Vectors. 2012;5:248.

14. Tokiwa T, Harunari T, Tanikawa T, Komatsu N, Koizumi N, Tung KC, Suzuki J, Kadosaka T, Takada N, Kumagai T, Akao N, Ohta N. Phylogenetic relationships of rat lungworm, Angiostrongylus cantonensis, isolated from different geographical regions revealed widespread multiple lineages. Parasitol Int. 2012;61:431-6.

15. Eamsobhana $\mathrm{P}$, Lim PE, Zhang H, Gan X, Yong HS. Molecular differentiation and phylogenetic relationships of three Angiostrongylus species and Angiostrongylus cantonensis geographical isolates based on a $66-\mathrm{kDa}$ protein gene of A. cantonensis (Nematoda: Angiostrongylidae). Exp Parasitol. 2010;126:564-9.

16. Eamsobhana P, Lim PE, Solano G, Zhang H, Gan X, Yong HS. Molecular differentiation of Angiostrongylus taxa (Nematoda: Angiostrongylidae) by cytochrome c oxidase subunit I (COI) gene sequences. Acta Trop. 2010;116:152-6.

17. Qvarnstrom Y, da Silva AC, Teem JL, Hollingsworth R, Bishop H, GraeffTeixeira C, da Silva AJ. Improved molecular detection of Angiostrongylus cantonensis in mollusks and other environmental samples with a speciesspecific internal transcribed spacer 1-based TaqMan assay. Appl Environ Microbiol. 2010;76:5287-9.

18. Liu CY, Zhang RL, Chen MX, Li J, Ai L, Wu CY, Zhu XQ, Lin RQ. Characterisation of Angiostrongylus cantonensis isolates from China by sequences of internal transcribed spacers of nuclear ribosomal DNA. J Anim Vet Adv. 2011;10:593-6.

19. Chen HT. Un nouveau nematode Pulmonaire, Pulmonema cantonensis, n. g., n. sp. des rats de Canton. Ann Parasitol Hum Comp. 1935;13:312-7.

20. Yokogawa S. A new species of nematode found in the lungs of rats, Haemostrongylus ratti sp. nov. Trans Nat Hist Soc Formosa. 1937;27:247-50.

21. Lv S, Zhang Y, Liu HX, Hu L, Yang K, Steinmann P, Chen Z, Wang LY, Utzinger J, Zhou XN. Invasive snails and an emerging infectious disease: results from the first national survey on Angiostrongylus cantonensis in China. PLoS Negl Trop Dis. 2009:3:e368

22. Yong HS, Eamsobhana P, Song SL, Prasartvit A, Lim PE. Molecular phylogeography of Angiostrongylus cantonensis (Nematoda: Angiostrongylidae) and genetic relationships with congeners using cytochrome b gene marker. Acta Trop. 2015;148:66-71.

23. Gasser RB, Chilton NB, Hoste $H$, Beveridge I. Rapid sequencing of rDNA from single worms and eggs of parasitic helminths. Nucleic Acids Res. 1993;21:2525-6.

24. Rodpai R, Intapan PM, Thanchomnang T, Sanpool O, Sadaow L, Laymanivong S, Aung WP, Phosuk I, Laummaunwai P, Maleewong W. Angiostrongylus cantonensis and A. malaysiensis broadly overlap in Thailand, Lao PDR, Cambodia and Myanmar: a molecular survey of larvae in land snails. PLoS One. 2016;11:e0161128.

25. Larkin MA, Blackshields G, Brown NP, Chenna R, McGettigan PA, McWilliam H, Valentin F, Wallace IM, Wilm A, Lopez R, Thompson JD, Gibson TJ, Higgins DG. Clustal W and Clustal X version 2.0. Bioinformatics. 2007;23:2947-8

26. Librado P, Rozas J. DnaSP v5: a software for comprehensive analysis of DNA polymorphism data. Bioinformatics. 2009;25:1451-2.

27. Posada D. jModelTest: phylogenetic model averaging. Mol Biol Evol. 2008;25:1253-6.

28. Ronquist F, Huelsenbeck JP. MrBayes 3: Bayesian phylogenetic inference under mixed models. Bioinformatics. 2003;19:1572-4.

29. Tamura K, Peterson D, Peterson N, Stecher G, Nei M, Kumar S. MEGA5: molecular evolutionary genetics analysis using maximum likelihood, evolutionary distance, and maximum parsimony methods. Mol Biol Evol. 2011;28:2731-9.

30. Maddison WP, Maddison DR. Mesquite: a modular system for evolutionary analysis. Version 275 (available at: http://mesquiteproject.org/. Accessed 10 Mar 2017.

31. Ellis RE, Sulston JE, Coulson AR. The rDNA of C. elegans: sequence and structure. Nucleic Acids Res. 1986;14:2345-64.

32. Conole JC, Chilton NB, Jarvis T, Gasser RB. Mutation scanning analysis of microsatellite variability in the second internal transcribed spacer (precursor ribosomal RNA) for three species of Metastrongylus (Strongylida: Metastrongyloidea). Parasitology. 2001;122:195-206.

33. LV S, Zhang YI, Steinmann P, Yang GJ Yang K, Zhou XN, Utzinger J. The emergence of angiostrongyliasis in the People's Republic of China: the 
interplay between invasive snails, climate change and transmission dynamics. Freshwater Biol. 2011;56:717-34.

34. $L i F H$, Zhou XM, Li YZ, Tao H. The occurrence of Angiostrongylus malaysiensis in Yunnan province. Chin J Parasit Dis Control. 1993;6:35.

35. Kobayashi N, Tamura K, Aotsuka T. PCR error and molecular population genetics. Biochem Genet. 1999;37:317-21.

36. Williams C, Ponten F, Moberg C, Soderkvist P, Uhlen M, Ponten J, Sitbon G Lundeberg J. A high frequency of sequence alterations is due to formalin fixation of archival specimens. Am J Pathol. 1999;155:1467-71.

37. Ganley AR, Kobayashi T. Highly efficient concerted evolution in the ribosomal DNA repeats: total rDNA repeat variation revealed by wholegenome shotgun sequence data. Genome Res. 2007;17:184-91.

38. Maslunka C, Gifford B, Tucci J, Gurtler V, Seviour RJ. Insertions or deletions (Indels) in the rrn 16S-23S rRNA gene internal transcribed spacer region (ITS) compromise the typing and identification of strains within the Acinetobacter calcoaceticus-baumannii (Acb) complex and closely related members. PLoS One. 2014;9:e105390.

39. Zhao Y, Tsang CC, Xiao M, Cheng J, Xu Y, Lau SK, Woo PC. Intragenomic internal transcribed spacer region sequence heterogeneity and molecular diagnosis in clinical microbiology. Int J Mol Sci. 2015;16:25067-79.

40. Powers TO, Todd TC, Burnell AM, Murray PC, Fleming CC, Szalanski AL, Adams BA, Harris TS. The rDNA internal transcribed spacer region as a taxonomic marker for nematodes. J Nematol. 1997;29:441-50.

41. Caldeira RL, Carvalho OS, Mendonca CL, Graeff-Teixeira C, Silva MC, Ben R, Maurer R, Lima WS, Lenzi HL. Molecular differentiation of Angiostrongylus costaricensis, A. cantonensis, and A. vasorum by polymerase chain reactionrestriction fragment length polymorphism. Mem Inst Oswaldo Cruz. 2003;98:1039-43

42. Jefferies R, Shaw SE, Willesen J, Viney ME, Morgan ER. Elucidating the spread of the emerging canid nematode Angiostrongylus vasorum between Palaearctic and Nearctic ecozones. Infect Genet Evol. 2010;10:561-8.

\section{Submit your next manuscript to BioMed Central and we will help you at every step:}

- We accept pre-submission inquiries

- Our selector tool helps you to find the most relevant journal

- We provide round the clock customer support

- Convenient online submission

- Thorough peer review

- Inclusion in PubMed and all major indexing services

- Maximum visibility for your research

Submit your manuscript at www.biomedcentral.com/submit
Biomed Central 